\title{
ASKING PRICE AND PRICE DISCOUNTS: THE STRATEGY OF SELLING AN ASSET UNDER PRICE UNCERTAINTY
}

\begin{abstract}
We consider fixed and asking price strategies in the context of selling an asset with Bernoullian updating of the seller's subjective probability of sale at a given price. The determination of optimal fixed, asking and endogenous reservation prices is discussed under risk-neutrality and expected utility maximisation. With risk-neutrality, the optimal asking price exceeds the optimal fixed price when the expected gain is a strictly concave function. The seller's choice between the fixed and the asking price strategies depends on several factors: the expected cost of haggling, price competition and the seller's attitude towards risk.
\end{abstract}

KEY WORDS: fixed price, asking price, price discounts, reservation price, risk attitude.

\section{INTRODUCTION}

The problem of optimal selling strategies has for some time been recognised as an interesting area for research. Following Telser (1973) and Rothschild (1974) on the buyer's problem of optimal search for the lowest prices, Riley and Zeckhauser (1983) and Rosenfield et al. (1983) analysed the optimal strategy for selling an asset. Although the motivations of the last two papers are different they have some common features. Both refer to a situation akin to an owner of an antique shop selling a valuable item over which the seller has in mind a subjective probability distribution of obtainable prices. After each bid from potential buyers, the seller decides whether or not to sell, weighing the possibility of a better bid against the cost of waiting. If the seller declines to sell the item, the buyer reveals the true value to the seller which leads 
the seller to a Bayesian updating of the subjective probability distribution. Ultimately, the item is sold when a buyer meets the updated reservation price.

The bulk of the literature in the 1970s and 1980s was devoted to this Bayesian updating process in the context of a sequence of bids. Bayesian updating models deal with the optimal selling strategy in a static framework. The seller faces a succession of bids within a single period and her subjective probability density function (pdf) of obtainable price from the buyers, $f(P)$, is defined with respect to the market in that period alone. The terms 'current' and 'future' denote positions in the queue, but they do not have a proper temporal connotation. Although a discount factor is sometimes used for successive encounters, the fact that waiting itself may affect the probability of selling a good is ignored.

Suppose a seller announces a fixed price, $P^{*}$. It may take a long time to sell the asset at that price. Given positive time-discounting, the buyer may be able to obtain a price discount. However, Riley and Zeckhauser, who make allowances for bargaining, argue that there is a strong case for a 'take-it-or-leave-it' pricing policy. Thanassoulis (2004), in contrast to the bulk of literature in the 1970s and 1980s, uses the economic theory of commodity bundling as the foundation of his paper, and shows that haggling may be part of the optimal selling strategy in the multi-product case. For example, package tour operators may offer clients a holiday at a reduced rate in a chosen country, the exact resort being unspecified and to be chosen later depending on the state of availability. By doing so, the operators may increase their profits. In the context of multi-product (substitutes) monopolists, an optimal selling strategy may involve haggling over the type of product (substitutes) to be delivered. In our paper, we are concerned with haggling over price in the context of selling a single asset. However, it is interesting to note that some of the results obtained in Thanassoulis are qualitatively similar to some of our results obtained by using a more traditional approach. 
If one intends to sell an asset, like a house or a used car, the seller's subjective probability distribution of the buyers' valuation of the asset (sometimes referred to as the distribution of the asset price) is derived from a significant amount of objective assessment. Before putting the asset on the market, the seller gathers information about the sale of similar items from newspapers, magazines and the internet, and seeks advice from surveyors and experts, so that she has an idea about the probability distribution of the asset-price for the foreseeable future. She is not so much worried about a mistake in predicting the pdf, as in determining the optimal asking price and reservation price. When a seller advertises a moderately expensive item (e.g. a house, car or yacht), she also specifies the asking price. Assuming (as we do) that the buyers make their bids sequentially, if the seller asks for the reservation price, she will not get a higher price. The gap between the asking price and the reservation price is called the price discount: the maximum reduction in price that the buyer can get. An analysis of strategies concerning asking prices and price discounts has important practical significance. For instance, in UK (excluding Scotland) and in many other countries, the housing market operates on the basis of asking prices. The asking price is generally different from the reservation price, known only to the seller. Once the seller announces a price for the asset, she may decide not to accept a price less than the announced price (fixed price strategy) or she may accept a price equal to, or above, a reservation price (asking price strategy). Once the asking price is announced, the seller must sell the property to the first buyer agreeing to buy the property at the asking price. There is an implicit assumption underlying the model presented here. The seller may have a pre-determined personal reservation price. Throughout, the paper we assume that the personal reservation price is less than the (endogenously determined) minimum acceptable price.

There is another important role of 'time' in a selling strategy. Given the asking price of the seller, the probability of a sale in period 1 (the current period) may be low, but if 
the seller is prepared to wait, the probability of sale over the period may increase significantly (a comparison is drawn with Bernoulli trials). In determining the optimal price for the asset, the seller should consider this inter-temporal aspect of her strategy. Horowitz's (1992) empirical work on asking and reservation prices for houses is based on a simple theoretical model, which has some similarities with ours: an expected utility maximising, risk-neutral seller with a specific utility function sets the asking price and the reservation price of a house. ${ }^{1}$ However, whilst Horowitz assumes the conditional probability of selling a house to be invariant with respect to time, in our model, the more a seller waits, the higher is the probability that she finds a buyer over the waiting period. This Bernoullian probability updating plays a crucial role in our paper. A discussion on the choice between the fixed price strategy and the asking price strategy together with the impact of non-risk-neutrality on the choice (two central themes in our paper), are also absent in Horowitz.

In this paper, we discuss the determination of the optimal asking price and the factors, which determine the extent of the price discount. The attitude of the seller towards risk has an important role to play here. Once we understand that the possibility of waiting may cause 'haggling', the range of haggling (between the asking and reservation prices) depends on the seller's attitude to risk (whether she is risk-averse, risk-neutral or risk-prone). Hence, it is not possible for the buyer to pre-determine the reservation price. This enforces the possibility of price-negotiations. Two important aspects of the selling strategy are discussed in detail: (i) the relationship between the optimal asking price, price discounts and the optimal fixed price, and (ii) the factors influencing the seller to adopt a 'fixed price' policy. The layout of the paper is as follows. Sections 2 and 3 introduce, respectively, the fixed and

\footnotetext{
${ }^{1}$ Naturally, the strategy of selling houses is a major area of interest. Albright (1977) uses the Bayesian updating technique to discuss the house selling strategy whilst empirical studies include Saas (1988) and Zuehlke (1987), amongst others.
} 
asking price strategies under risk-neutrality and analyses them in each case. Section 4 considers the question of when to haggle. Section 5 examines the effect of allowing more general risk attitudes to the relative attractiveness of the two selling strategies. Section 6 contains concluding remarks.

\section{OPTIMAL FIXED PRICE: A RISK-NEUTRAL SELLER}

Suppose the seller of an asset has a continuous (truncated) subjective probability distribution of buyers' valuations of the asset in mind with the domain $I=[a, b], b>a>0$. If the seller sets the price at $a$, the asset should be sold immediately (in period 1). The price ' $b$ ' represents the upper limit of the prices for which the asset may perceivably be sold. In the context of a probability distribution, $P$ denotes the buyers' evaluation (demand price), whilst in terms of pricing the asset, $P$ refers to the seller's asking price. We assume that the subjective pdf of the asset price in each period remains unchanged over time. In other words, the seller expects the nature of the market to remain the same over the foreseeable future. The seller's subjective distribution of buyers' valuations may be affected by various factors in the future, e.g. a long period of waiting or new information regarding the buyers' valuations. When a revision is required, the seller will recalculate and reset the asking price. However, the seller is assumed to be myopic in the sense that the subjective distribution for all periods in the future is based on current information: the optimisation problem faced by the seller in this model is conditioned by her present information of the market. This limitation stems from her inability to predict future changes in the market or the length of time for which the asset will remain unsold in the market.

We denote the seller's subjective pdf of the buyers' valuations of the asset as $f(P)$. We define, $F(P)=\int_{a}^{P} f(\pi) \mathrm{d} \pi$, $F(a)=0$ and $F(b)=1$. The probability of the asset being sold within any one period at price $P$ is $p=1-F(P)$. Given the seller's fixed price $P$, the probability of selling the asset 
in period $N$ is $(1-p)^{N-1} p$. If the asset is sold at price $P$ in period $N(N=1, \ldots, \infty)$, the discounted present-day value of the asset is $P(1+r)^{1-N}$, where $r<1$ is the market interest rate. We assume the capital market to be perfect. If the seller announces a fixed price $P$, the expected return from the sale is,

$$
\begin{aligned}
E & =\sum_{N=1}^{\infty} P(1+r)^{1-N}(1-p)^{N-1} p \\
& =p P \sum_{N=1}^{\infty}\left(\frac{1-p}{1+r}\right)^{N-1}=P \frac{(1+r) p}{r+p} .
\end{aligned}
$$

Since, $p \leq 1, P \geq E$. Note, if the seller adopts a fixed price strategy, the fixed price $P$ must be such that $E \geq a$. To begin with, we assume that the seller is risk-neutral. A risk-neutral seller maximises the expected gain $E$. If $P^{*}$ is the optimal fixed price, $E^{*}$ is the expected value from selling the asset at this price. The properties of $E$ turn out to be of importance. In particular, we note that $E$ may not be a concave function of $P$ everywhere.

LEMMA 1. $E$ is a strictly concave function of $P$ everywhere if and only if,

$$
2 \frac{\mathrm{d} p}{\mathrm{~d} P}+P \frac{\mathrm{d}^{2} p}{\mathrm{~d} P^{2}}-\frac{2 P}{r+p}\left[\frac{\mathrm{d} p}{\mathrm{~d} P}\right]^{2}<0 .
$$

Proof of Lemma 1. Differentiating $E$ twice with respect to $P$ and setting it less than 0 , we get,

$$
2 r \frac{\mathrm{d} p}{\mathrm{~d} P}+P \frac{r(r+p)^{2} \frac{\mathrm{d}^{2} p}{\mathrm{~d} p^{2}}-2 r(r+p)\left[\frac{\mathrm{d} p}{\mathrm{~d} P}\right]^{2}}{(r+p)^{2}}<0 .
$$

Further simplifying we obtain (2).

QED

Since $\mathrm{d} p / \mathrm{d} P=-f(P)<0$, a sufficient condition for $E$ being a strictly concave function of $P$ everywhere within any arbitrary non-negative domain $I$ is that $\mathrm{d}^{2} p / \mathrm{d} P^{2}=-f^{\prime}(P)$ is non-positive everywhere in the domain $I$. In other words, 
if $f^{\prime}(P)=0$ (a uniform distribution) or $f^{\prime}(P)>0 \forall P, E$ is strictly concave everywhere. The condition $f^{\prime}(P)>0$ implies that $F(P)$, the probability of not finding a customer in a particular period, increases at a faster rate as $P$ increases. To require $E$ to be concave over any arbitrary non-negative domain is asking a lot. Hence, the class of distributions which comply with the condition is very limited.

However, the concavity of $E$ over the price range $[a, b]$ depends not only on the nature, and parameters, of the (truncated) distribution function but also on the boundary values, $a$ and $b$ (both are non-negative and $b$ is finite). $E$ may be concave for almost any well-known distribution (e.g. exponential, Gamma and Beta) when truncated over a suitable price range $[a, b]$. Without any loss of generality we can always linearly transform $P$ such that, $a=0$ and $b$ equals any arbitrary positive value, depending on the unit of measurement chosen. To illustrate the above argument, let us consider the price range $[a, b]=[0,0.8]$ and a truncated exponential distribution function, $F(P)=1-\mathrm{e}^{-P}+\mathrm{e}^{-b} P / b$, with the density function, $f(P)=\mathrm{e}^{-P}+\mathrm{e}^{-b} / b$. From lemma $1, E$ is concave if $(r+p)\left[\mathrm{d}^{2} p / \mathrm{d} P^{2}\right]-2[\mathrm{~d} p / \mathrm{d} P]^{2}<0$ (a sufficient condition). Since, by assumption, $r<1, r+p<2$. It is easy to check that $\frac{\mathrm{d}^{2} p}{\mathrm{~d} P^{2}}=$ $\mathrm{e}^{-P}<2 \frac{\mathrm{e}^{-P} \mathrm{e}^{-b}}{b}<\left[\frac{\mathrm{d}_{p}}{\mathrm{~d} P}\right]^{2}$ for $b \leq 0.8$.

In general, for a unimodal density function, let $M(>a)$ be the mode. From lemma $1, E$ is concave when $f^{\prime}(P) \geq 0$. It is easy to see that possible values of $b(>M)$ exist such that at $b, \operatorname{Mod}\left(\mathrm{d}^{2} p / \mathrm{d} P^{2}\right)=f^{\prime}(P)<[f(P)]^{2}$. In other words, the concavity of $E$ depends on the parameters $a, b$ and the nature, as well as the parameters, of the truncated density (distribution) function. This applies to any well-known unimodal distribution function defined over $P>0$.

PROPOSITION 1. For a risk-neutral seller, (i) the optimal fixed price $P^{*}$ must be less than $b$, (ii) if $P^{*}>a, P^{*}$ satisfies the implicit (open loop) relationship,

$$
P^{*}=\frac{p(p+r)}{r f\left(P^{*}\right)} .
$$


Proof of Proposition 1. (i) When $P=b$ (i.e. $p=0$ ), $E=0$. For $P=a, E=a$. Obviously, $P=a$ is a better choice than $P=$ $b$. Therefore, the optimal fixed price $P^{*}$ must be less than $b$. (ii) A seller adopting the fixed price strategy sets $P^{*}$ to maximise the expected earning, $E$. Differentiating $E$ with respect to $P$, setting $\mathrm{d} E / \mathrm{d} P=0$, and noting $\mathrm{d} p / \mathrm{d} P=-f(P)$, we get,

$$
0=p+P \frac{f(P) p-f(P)(p+r)}{p+r},
$$

which yields (3).

QED

If (2) is satisfied, the optimal fixed price is unique. The implicit relationship (3) is useful in calculating the optimum fixed price. For example, suppose a property in the price range $I=[\$ 50 k, \$ 60 k]$ is to be sold, $f(P)$ is a uniform pdf and the market interest rate is $r=0.05$. In this case, (3) yields, $0.05(10)^{-1} P=[(60-P) / 10]^{2}+0.05[(60-P) / 10]$, or, $P^{2}-$ $121 P-3630=0$. Solving the quadratic equation for $P$, in the relevant range $[\$ 50 k, \$ 60 k]$, we get $P^{*}=\$ 55 k$ with expected yield, $E^{*}=\$ 52.5 k$.

The fixed price strategy is usually a sub-optimal strategy. Barring any special reason, if a buyer places a firm bid somewhere between the fixed price $(P)$ and the expected return $(E)$, her bid should be accepted by the risk-neutral seller. Riley and Zeckhauser (p. 267) note: 'Antique stores may post prices and suggest on casual inquiry that they are fixed. These prices may be cut, or extras thrown in, for sophisticated buyers.' Hence, in order to make the fixed price strategy work, the seller may have to appoint a selling agent. This is a strategy to keep the buyer at arm's length. However, if a seller is selling several assets of a similar nature, she may refuse any pricediscount in an attempt to convince the market that, for her, 'fixed price' means fixed price. She hopes that this will establish a reputation and help her avoid any future cost of haggling with other assets. 


\section{OPTIMAL ASKING PRICE: THE RISK-NEUTRAL SELLER}

In this section we consider the asking price strategy. If $P$ is the asking price, the risk-neutral seller may wish to accept a price less than $P$. The seller will not accept a price worth less than $E$, because her (discounted) expected return would be higher if she is prepared to wait. However, her reservation price may be more than $E$. We denote the reservation price as $M$. Given $P$ and $M$, the expected yield from the asking price strategy is given by,

$$
\begin{aligned}
V= & E+\sum_{N=1}^{\infty} \int_{M}^{P}\left[(\pi-E)(1+r)^{1-N} \frac{f(\pi)}{\int_{M}^{P} f(\pi) \mathrm{d} \pi} \mathrm{d} \pi\right] \\
& {\left[\int_{M}^{P} f(\pi) \mathrm{d} \pi\right]\left[1-\int_{M}^{P} f(\pi) \mathrm{d} \pi\right]^{N-1} } \\
= & E+\int_{M}^{P}[(\pi-E) f(\pi)] \mathrm{d} \pi \frac{(1+r)}{r+\int_{M}^{P} f(\pi) \mathrm{d} \pi} \\
= & E+\int_{M}^{P}[(\pi-E) f(\pi)] \mathrm{d} \pi \frac{(1+r)}{r+\phi} .
\end{aligned}
$$

where, $\phi=\int_{M}^{P} f(\pi) \mathrm{d} \pi$. Equation (4) needs explanation. The asset will be sold in one of two ways. First, a buyer may agree to pay the asking price $P$. Given bids are assumed to arrive sequentially, no buyer needs to offer a price more than $P$. The expected return from such an event over time is $E$, the first term in (4). The second term in (4) appears due to the fact that a potential buyer may negotiate a price $\pi(M \leq \pi \leq$ $P)$. This is the expected additional gain from the flexibility of the asking price policy over the expected yield from the fixed price policy. The expected discounted surplus over $E$ is calculated by using conditional probabilities, hence we divide $f(\pi)$ by $\phi$, and the probability weights add up to 1 . The risk-neutral seller will set $P$ and $M$ in such a way that the expected yield $V$ is maximised. Since $P$ lies in a compact interval of the real line and $V$ is continuous, the solution will exist. The interior solution must satisfy two non-linear constraints $\partial V / \partial P=$ 
0 and $\partial V / \partial M=0$. Explicit (closed loop) solutions for $P$ and $V$ in the general case can not be obtained.

DEFINITION 1. The pure price discount (ppd) is defined as the difference between the asking price and the reservation price (minimum acceptable price) of a risk-neutral seller.

We use the adjective 'pure' in order to distinguish ppd from the price discount acceptable to a risk-averse or risk-loving seller, which will be shown later as a sum of ppd and a risk-attitude factor. Except for the extreme case, $P=a$, the seller will accept a discount on the asking price, i.e. the buyer should always bargain.

PROPOSITION 2. For any asking price $P$, let the pure price discount be denoted by $\delta(P)$. Then,

$$
\delta(P)=P \frac{r(1-p)}{r+p}-\frac{\int_{M}^{P}(\pi-E) f(\pi) \mathrm{d} \pi}{r+\phi} .
$$

Proof of Proposition 2. For a given value of $P$, the value of $E$ is known by (1). First we prove that given any asking price $P$, the corresponding optimal reservation price $M$ satisfies the implicit relationship (6),

$$
\begin{aligned}
0= & \partial V / \partial M=-\frac{(M-E) f(M)(1+r)}{r+\phi} \\
& +\frac{f(M)(1+r) \int_{M}^{P}(\pi-E) f(\pi) \mathrm{d} \pi}{(r+\phi)^{2}},
\end{aligned}
$$

which yields,

$$
M=E+\frac{\int_{M}^{P}(\pi-E) f(\pi) \mathrm{d} \pi}{r+\phi} .
$$

Since $\delta(P)=P-M=(P-E)-(M-E)$, Proposition 2 follows by calculating $(P-E)$, using (1) and (6).

QED

The above proposition and the following remarks are valid for any arbitrary asking price and, consequently, at the optimal asking price. 
REMARK 1. From (6) it is clear that for any asking price, $P$, the reservation price, $M$ is more than the expected yield $E$, if $P$ is treated as given.

REMARK 2. Given any asking price $P$, the reservation price $M$ is likely to be significantly closer to $V$ than $E$. This can be shown as follows. From (4) and (6), by eliminating the expression involving integrals and simplifying, we get,

$$
\frac{V-M}{M-E}=r .
$$

The interest rate is usually a small percentage. Hence, by (7), $M$ should be significantly closer to $V$ than $E$.

REMARK 3. If $\partial E / \partial P<0$ in the neighbourhood of the optimal asking price, locally $\partial M / \partial P<0$ for $P$ and $M$ satisfying the condition $\partial V / \partial M=0$.

To see this, differentiating Equation(7) with respect to $P$ and rearranging terms yields, $\partial V / \partial P+r \partial E / \partial P=(1+r) \partial M / \partial P$. At the optimal asking price, $\partial V / \partial P=0$. Hence, the result follows. This result will be useful later on.

PROPOSITION 3. Given an asking price $P, a<P<b$, an increase in the interest rate will reduce the reservation price $M$ and consequently the pure price discount $\delta(P)$ will increase.

Proof of Proposition 3. Partially differentiating $E$ with respect to $r$, with $P$ given, we get,

$$
\partial E / \partial r=P \frac{p^{2}-p}{(r+p)^{2}}<0 .
$$

Partially differentiating $M$ with respect to $r$, using (1) in (6), we obtain,

$$
\begin{aligned}
& \frac{\partial M}{\partial r}=\frac{\partial E}{\partial r}+\frac{-(M-E) f(M) \frac{\partial M}{\partial r}-\frac{\partial E}{\partial r} \phi}{r+\phi}-\frac{\int_{M}^{P}(\pi-E) f(\pi) \mathrm{d} \pi}{(r+\phi)^{2}}, \\
& \frac{\partial M}{\partial r}\left[1+\frac{(M-E) f(M)}{r+\phi}\right]=\frac{\partial E}{\partial r}\left(1-\frac{\phi}{r+\phi}\right) \\
& -\frac{\int_{M}^{P}(\pi-E) f(\pi) \mathrm{d} \pi}{(r+\phi)^{2}} .
\end{aligned}
$$

Originally published in Theory and Decision (2007) 62:281-301 (c) Springer 
The coefficient of $\partial M / \partial r$ is positive and both terms on the right-hand side are negative. Hence $\partial M / \partial r<0$ and for any given $P, \delta(P)=P-M$ must increase with an increase in the interest rate $r$.

QED

It is a common belief that sellers set asking prices above the optimal fixed price because sellers know that potential buyers will haggle to buy the asset. If the asking price is lower, it may seem that the seller can always set the asking price equal to the higher fixed price and keep the same reservation price so that they can sell the asset to the customers offering to buy the asset at the reservation price or more. This is not correct. A higher asking price affects the inter-temporal probabilities, i.e. it affects the probability of the future event where the asset may be sold at a price less than the asking price. For example, the probability of selling an asset in the period $N$ is $p_{N}=p(1-p)^{N-1}$. Differentiating with respect to $p$, we can see that $\mathrm{d} p_{N} / \mathrm{d} p>0$ if $1-p>(N-1) p$ and vice versa.

Next we prove that if (2) in Lemma 1 holds, the optimal asking price is greater than the optimal fixed price.

PROPOSITION 4. If $E$ is a strictly concave function of $P$ and $r<1$, the optimal asking price will be greater than the optimal fixed price.

Proof of Proposition 4. At the optimal asking price,

$$
\begin{aligned}
\frac{\partial V}{\partial P}= & \frac{\partial E}{\partial P}+\frac{(1+r)\left[(P-E) f(P)-\frac{\partial E}{\partial P} \phi\right]}{r+\phi} \\
& -\frac{(1+r) f(P) \int_{M}^{P}(\pi-E) f(\pi) \mathrm{d} \pi}{(r+\phi)^{2}}=0 .
\end{aligned}
$$

Therefore, using (6),

$$
\frac{\partial E}{\partial P}\left[\frac{(1+r) \phi}{r+\phi}-1\right]=\frac{(1+r) f(P)}{r+\phi}[(P-E)-(M-E)] .
$$

Given $\phi<1$ and $r<1$, the coefficient of $\partial E / \partial P$ is negative. On the right-hand side, the term within the bracket equals 


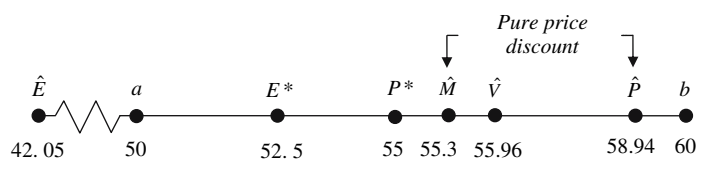

Figure 1. An illustration of the optimal asking price, $\hat{P}$, fixed price, $P^{*}$, and the pure price discount, $\delta(P)$, in $\$ 000$.

$P-M>0$. Hence, at the optimal asking price $\partial E / \partial P$ must be strictly negative. If $E$ is a strictly concave function of $P$, the optimal asking price, which maximises $V$, must be greater than the optimal fixed price, which maximises $E$.

QED

To illustrate, consider the example of the property seller introduced in Section 2, for which the pdf of offers is uniform and bounded between $\$ 50 \mathrm{k}$ and $\$ 60 \mathrm{k}$. Imposing these assumptions in (6), yields, $M=E+\{(P+M)(P-M)-2 E$ $(P-M)\} /\{20[r+(P-M) / 10]\}$. Substituting into (7), using (1) and re-arranging, yields an expression for $V$ in terms of $P$. Maximising with respect to $P$ yields $\hat{P}=58.94$ (where $\hat{P}$ is the optimal asking price). Figure 1 reports the optimum price, $\hat{P}$, expected value, $\hat{V}$, and reservation price, $\hat{M}$, for the asking price strategy along with the relevant values for the optimum fixed price strategy (derived in Section 2). Note, since we are assuming a uniform distribution, $E$ is strictly concave, and the optimal asking price is greater than the optimal fixed price, as expected.

From proposition 4, we get,

$$
P=M+\theta, \text { where } \theta=\frac{\partial E}{\partial P} \frac{r}{1+r} \frac{(\phi-1)}{f(P)} .
$$

When $E$ is a concave function of $P$, the magnitudes as well as the signs of $\partial \theta / \partial P$ and $\partial \theta / \partial r$, at the optimal $P$, determine the effects of a change in the interest rate on the asking price, the minimum acceptable price and the price discount. In general, the signs of the two derivatives depend on the parameters of the model. Note, at the optimal solution, when $M$ is optimally adjusted, $\theta=\partial(P)$. Figure 2, illustrates the case of $\partial \theta / \partial P<1$ given $r$ and $\partial \theta / \partial r>0$ given $P$. The curve $M M$ represents the 


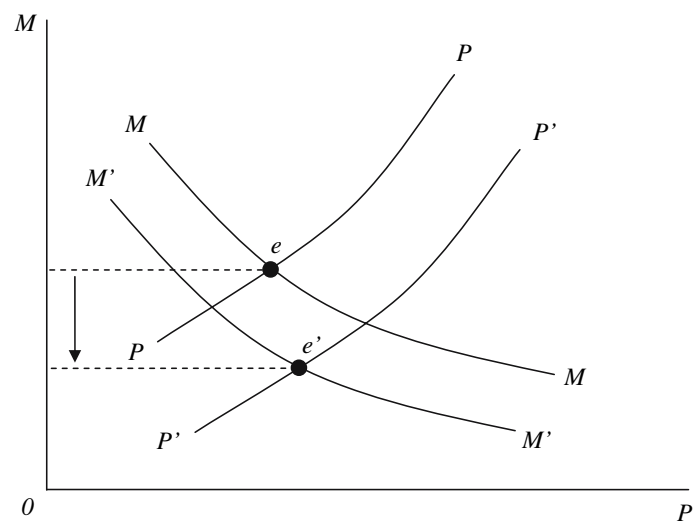

Figure 2. The effect of an increase in the interest rate on the reservation price (M) when $\partial \theta / \partial P<1$ and $\partial \theta / \partial r>0$.

combination of $M$ and $P$ for $\partial V / \partial M=0$. Since $\partial E / \partial P<0$ at the optimal solution when $E$ is concave, by remark 3 of proposition 2, $M M$ has a negative slope in the neighbourhood of the optimal solution. By proposition 3, $M M$ shifts downwards to $M^{\prime} M^{\prime}$ with an increase in $r$. The curve $P P$ represents the combinations of $M$ and $P$ for $\partial V / \partial P=0$. When $\partial \theta / \partial P<1$ for a given $r, P P$ has a positive slope. The $P P$ curve shifts downwards to $P^{\prime} P^{\prime}$ when $\partial \theta / \partial r>0$ because for any particular value of $P$, the value of $M$ will be smaller by Equation (8). The optimal solution moves from $e$ to $e^{\prime}$. The minimum asking price in this case is lower. However, the effects on $P$ and $\partial(P)$ depend on the relative extent of shifts in $P P$ and $M M$.

\section{WHEN TO HAGGLE AND WHEN TO HOLD FIRM: ASKING PRICE VERSUS FIXED PRICE}

When should the seller adopt a fixed price policy and under what circumstances should the seller allow potential buyers to haggle? There is evidently a cost of haggling to the seller in terms of the value of the time and energy spent. For simplicity, we assume that the expected cost of haggling is constant, 
$c$. From an announced price $P$, the seller expects to gain $G=$ $V-E$, from adopting the asking price strategy rather than treating $P$ as a fixed price. The expected net gain from the asking price strategy is expressed as,

$$
G=V-E=\frac{(1+r)}{r+\phi} \int_{M}^{P}[(\pi-M) f(\pi)] \mathrm{d} \pi .
$$

Given that $E^{*}$ denotes the expected yield associated with the optimal fixed price $P^{*}$, the risk-neutral seller will allow bargaining if $V(P)-c>E^{*}$, i.e. $E-E^{*}+G>c$, and will adopt a fixed price policy if $E-E^{*}+G<c$. It is worthwhile analysing (9) in detail. Since $P \geq V \geq M \geq E$, if the difference between $P$ and $E$ is very small, $G$ will be very small and the seller is likely to adopt a 'fixed price' policy. Note, $E^{*} \geq E$ because $E^{*}$ is the maximum value of $E$ in (1). There are two interesting situations when the difference between $P$ and $E$ is likely to be very small. First, note that $P-E=P(1-p) r /(p+r)$ and $(1-p) r /(p+r) \leq 1$. Hence, if the asking price $P$ is very small, the difference between $P$ and $E$ is very small. Usually, for inexpensive items, the difference between the asking price and the minimum acceptable price is very low so that $G$ is very small and the seller is not likely to negotiate prices on such items. In the case of expensive items, the gap between the asking price and the minimum acceptable price may be wide. In this case $G$ may be large enough, such that $E-E^{*}+G>c$ and the seller adopts the asking price policy. In real life, bargaining is quite customary in selling expensive assets. Second, suppose the asset is being sold in a fairly competitive market such that the price range, $b-a$, is very small. If $P$ is the average asking price, an arbitrarily small price discount on $P$ will lead to an immediate sale of the asset, i.e. $M$ is arbitrarily close to $P$. In such cases, $G$ is very small and the sellers are likely to adopt a 'fixed price' strategy.

PROPOSITION 5. The following observations establish a link between the fixed price strategy and the asking price strategy: (i) The asking price strategy emulates the fixed price strategy 
when, at the optimal asking price $P, \partial E / \partial P$ is arbitrarily close to 0 . (ii) If the optimal asking price is $P=a$, the seller adopts a fixed price strategy. (iii) If the optimal asking price is $P=b$, the optimal price discount, $\partial(P)>0$ and $M \geq P \phi$, which is the expected value of the buying offers in the current period.

Proof of Proposition 5. Since at the optimal solution, $\theta=\partial(P)$, we can rewrite Eq. (8) as,

$$
\partial(P)=\frac{\partial E}{\partial P} \frac{r}{1+r} \frac{(\phi-1)}{f(P)}=\left[\frac{p r}{r+p}-\frac{P f(P) r^{2}}{(r+p)^{2}}\right] \frac{\phi-1}{f(P)} .
$$

(i) If the optimal asking price is arbitrarily close to the optimal fixed price (as in the case of an almost perfectly competitive market with a negligible variance in $P), \partial E / \partial P$ is close to 0 at the optimal asking price. As $\partial E / \partial P \rightarrow 0, \partial(P) \rightarrow 0$ and the optimal asking price strategy emulates the fixed price strategy. Hence, the fixed price strategy may be treated as a special case of the asking price strategy. (ii) When the optimal asking price is $P=a$, the seller expects to sell the asset in period 1 at the asking price. Hence, $\partial(P)=0$. (iii) $M<b$, otherwise $V=0$. Therefore, $\partial(P)>0$. When $P=b$, the probability of sale at that price is 0 . Substituting $p=0$ in the above equation we get $\partial(P) \leq P(1-\phi)$. This implies $M \geq P \phi$. QED

\section{ATTITUDE TOWARDS RISK AND THE PRICE DISCOUNT}

In the last two sections, we analysed the behaviour of a riskneutral seller maximising the expected return from the sale of an asset. In this section we drop the assumption of risk-neutrality. In the context of the fixed price strategy, if the fixed price is $P^{*}$, the expected utility from sale is,

$$
\begin{aligned}
& E(U)=\sum_{N=1}^{\infty} U\left(P^{*}\right)(1+\rho)^{1-N} p(1-p)^{N-1} \\
& =\sum_{N=1}^{\infty} U\left(P^{*}\right) p\left[\frac{1-p}{1+\rho}\right]^{N-1}=U\left(P^{*}\right) \frac{p(1+\rho)}{p+\rho}\left(U \geq 0, U^{\prime}>0\right) .
\end{aligned}
$$

Originally published in Theory and Decision (2007) 62:281-301 (c) Springer 
The rate at which future utilities are discounted is given by $\rho$. Except in the case of $P^{*}=a$ (i.e. $\left.p=1\right), E(U)<U\left(P^{*}\right)$. This implies that whatever the seller's attitude towards risk (risk-averse or risk-prone), the possibility of a price discount under the asking price strategy always exists. The certainty equivalent price of $E(U)$ is $\tilde{P}$, where $U(\tilde{P})=E(U)$. Under the fixed price strategy, $P$ will be set in such a way that $E(U)$ is maximised. In the context of the asking price strategy, the expected yield is given by,

$$
\begin{aligned}
V_{U}= & E(U)+\sum_{N=1}^{\infty} \int_{M}^{P}[U(\pi)-E(U)](1+\rho)^{1-N} \frac{f(\pi)}{\int_{M}^{P} f(\pi) \mathrm{d} \pi} \mathrm{d} \pi \\
& {\left[\int_{M}^{P} f(\pi) \mathrm{d} \pi\right]\left[1-\int_{M}^{P} f(\pi) \mathrm{d} \pi\right]^{N-1} } \\
= & E(U)+\int_{M}^{P}[U(\pi)-E(U)] f(\pi) \mathrm{d} \pi \frac{(1+\rho)}{\rho+\phi} .
\end{aligned}
$$

The expected utility maximising seller will set the asking price $P$ and the reservation price $M$ at levels which maximise $V_{U}$. The price discount, $\tilde{\delta}(P)$, is the difference between the asking price and the reservation price $(P-M)$, as defined in Section 2 , but in the context of different possible attitudes to risk. The expected net gain from the asking price strategy over the fixed price strategy, for any given $P$, is,

$$
G_{U}=V_{U}-E(U)=\int_{M}^{P}[U(\pi)-E(U)] f(\pi) \mathrm{d} \pi \frac{(1+\rho)}{\rho+\phi} .
$$

If the seller is risk-averse (risk-prone), by the Arrow-Pratt criterion, ${ }^{2}$ we know that the expected utility from uncertain returns is less (more) than the utility from the expected return. The two are equal under risk-neutrality. Further note, as previously discussed, $E(U)$ is obtained from a fixed price scenario.

\footnotetext{
${ }^{2}$ See Pratt (1964). For a discussion of the generalised Arrow-Pratt measures of risk aversion see Biswas (1983).
}

Originally published in Theory and Decision (2007) 62:281-301 (c) Springer 
PROPOSITION 6. Given any asking price $P$, the price discount, $\tilde{\delta}(P)$, on the asking price is more (less) than the pure price discount, $\delta(P)$, if the seller is risk-averse (risk-prone).

Proof of Proposition 6. Partially differentiating $V_{U}$ with respect to $M$ and setting $\partial V_{U} / \partial M=0$, we get,

$$
\begin{aligned}
U(M) & =E(U)+\frac{\int_{M}^{P}[U(\pi)-E(U)] f(\pi) \mathrm{d} \pi}{\rho+\phi} \\
& =E(U)+\frac{\phi}{\rho+\phi} \int_{M}^{P}[U(\pi)-E(U)] \frac{f(\pi)}{\phi} \mathrm{d} \pi .
\end{aligned}
$$

Note, $\int_{M}^{P} f(\pi) / \phi \mathrm{d} \pi=1$. In fact, $f(\pi) / \phi$ is a conditional pdf. Hence, the above is rewritten as,

$$
U(M)=E(U)\left[1-\frac{\phi}{\rho+\phi}\right]+\frac{\phi}{\rho+\phi} \int_{M}^{P} U(\pi) \frac{f(\pi)}{\phi} \mathrm{d} \pi .
$$

Let $\bar{\pi}=\int_{M}^{P} \pi f(\pi) / \phi \mathrm{d} \pi$. It is well known that $\int_{M}^{P} U(\pi) f(\pi) / \phi \mathrm{d} \pi$ is equal to $U(\bar{\pi})$ when $U$ is a linear function (risk-neutrality), less than $U(\bar{\pi})$ if $U$ is concave and greater than $U(\bar{\pi})$ if $U$ is convex. Since $U(M)$ is a monotonically increasing function of $M$, it implies that $M_{1}<M_{0}<M_{2}$, where $M_{0}, M_{1}$ and $M_{2}$ are reservation prices under risk-neutral, risk-averse and riskprone attitudes. Since $\tilde{\delta}(P)=P-M$, for any given price $P$ the proposition follows immediately.

QED

In the case of a risk-neutral seller, the pure price discount, $\delta(P)=P-M_{0}$. Therefore, in the case of non-risk-neutrality, the price discount is augmented by a risk attitude factor, which is positive in the case of risk aversion and negative in the case of risk-prone sellers.

PROPOSITION 7. Given the asking price $P>a$, the seller is more likely to adopt a fixed price policy if she is risk-prone.

Proof of Proposition 7. The price discount obtainable from a risk-prone seller is $\tilde{\delta}(P)=P-M_{2}=\left(P-M_{0}\right)-\left(M_{2}-M_{0}\right)$. By proposition $6, M_{2}-M_{0}$ is positive if the seller is riskprone and consequently $P-M_{2}$ is smaller. The more riskprone the seller is, the smaller is $P-M_{2}$. If the seller is a 
highly risk-prone person, $P-M_{2}$, and consequently $G_{U}$ in (12) is very small. Suppose $P^{*}$ and $E^{*}(U)$ are the optimal fixed price and the associated expected utility. Let $P$ and $V_{U}$ be the optimal asking price and the associated expected utility. Since $V_{U}=E(U)+G_{U}$, the seller will adopt a fixed price policy if, $V_{U}-H=E(U)+G_{U}-H<E^{*}(U)$, where, $H$ is the expected utility-cost of haggling. Since, $E(U)$ is maximised at $P^{*}, E(U) \leq$ $E^{*}(U)$. We have noted, when the seller is highly risk-loving, $G_{U}$ is very small. Therefore, the seller may prefer the fixed price strategy to the asking price strategy.

QED

\section{CONCLUSION}

We have compared the asking price and fixed price strategies for selling an asset in an uncertain market. The asking price strategy requires simultaneous determination of the optimal asking price and the minimum acceptable price (reservation price). The gap between the two is the price discount: the maximum reduction in price which the buyer may obtain through bargaining. The asking price and the reservation price are affected by the seller's attitude towards risk. The attitude towards risk also affects the choice between a fixed price strategy and an asking price strategy. We identified three primary reasons for the adoption of a fixed price strategy: (i) the item is so inexpensive that it is not worth negotiating the price; (ii) the market for the asset is highly competitive; (iii) the seller is highly risk-prone so that she would rather stick to the fixed price than sell immediately with a price discount. Whilst it is theoretically possible that the optimal asking price may be less than the optimal fixed price, we show that if the expected return function is strictly concave then the optimal asking price must exceed the optimal fixed price. The existing literature on optimal selling strategies mainly explores the hypothetical situation in which the seller receives a sequence of bids which she may or may not accept. In light of the past revelations the seller revises her reservation price. We have 
mentioned a few such papers in the introduction. Our paper takes a different strand in trying to analyse the more common strategy of setting an asking price and the determination of price discounts associated with it. An analysis of the asking price strategy in an inter-temporal context and with the relaxation of the assumption of risk-neutrality is an important step towards an understanding of the theory of selling strategies. This paper proves some useful results towards that understanding.

\section{ACKNOWLEDGEMENTS}

We are grateful to the referee of this journal for helpful suggestions which significantly improved the quality of presentation. We also wish to thank Michael Nolan for useful discussions in the development of this paper. Regarding errors, the usual caveats apply.

\section{REFERENCES}

Albright, S.C. (1977), A Bayesian approach to a generalized house selling problem, Management Science 24, 432-440.

Biswas, T. (1983), A note on the generalised measures of risk aversion, Journal of Economic Theory 29, 347-352.

Horowitz, J.L. (1992), The role of the list price in housing markets: Theory and an econometric model, Journal of Applied Econometrics 7, 115-129.

Pratt, J.W. (1964), Risk aversion in the small and in the large, Econometrica 32, 122-136.

Riley, J. and Zeckhauser, R. (1983), Optimal selling strategies: When to haggle and when to hold firm, Quarterly Journal of Economics 98, 267-289.

Rothschild, M. (1974), Searching for the lowest price when the distribution of prices is unknown, Journal of Political Economy 82, 689-711.

Rosenfield, D.B., Shapiro, R.D. and Butler, D.A. (1983), Optimal strategies for selling an asset, Management Science 29, 1051-1061.

Saas, T.R. (1988), A note on optimal price cutting behaviour under Demand uncertainty, Review of Economics and Statistics 70, 336-339.

Telser, L.G. (1973), Searching for the lowest price, American Economics Review (Papers and Proceedings) 63, 40-49. 
Thanassoulis, J. (2004), Haggling over substitutes, Journal of Economic Theory 118, 217-245.

Zuehlke, T.W. (1987), Duration dependence in the housing market, Review of Economics and Statistics 69, 701-709.

Address for correspondence: Tapan Biswas, Centre for Economic Policy, University of Hull, Cottingham Road, Hull, HU6 7RX, UK. E-mail: t.k.biswas@hull.ac.uk

Jolian Mchardy, Department of Economics, University of Sheffield, 9 Mappin Street, Sheffield, S1 4DT, UK. E-mail: J.mchardy@sheffield.ac.uk 